\title{
Effect of charge on growth of droplets with ionic condensation nuclei
}

\author{
GUO PingSheng $^{1 *} \&$ LIU HaiLi ${ }^{2}$ \\ ${ }^{1}$ Physics Science and Technology Institute of Guangxi Normal University, Guilin 541004, China; \\ ${ }^{2}$ Department of Physics and Information Engineering, Hunan Institute of Humanities, Science and Technology, Loudi 417000, China
}

Received July 5, 2010; accepted October 22, 2010; published online May 12, 2011

\begin{abstract}
A growth kinetics model of droplets with ionic condensation nuclei based on the chemical potential and the kinetic equation of mass transfer is established. The model is simplified and the effect of charge on droplet growth is examined. The theoretical results show that the critical radius for droplet growth with an ionic condensation nucleus is less than that of those without an ionic nucleus. Furthermore, our results also indicate that if the initial droplet with an ionic nucleus has a radius shorter than the critical radius, the droplet will not vanish, but will reach a steady-state radius. As the ionic charge increases, the critical radius for droplet growth will decrease and the corresponding steady-state droplet radius will increase. In addition we show that once a critical charge is reached, all droplets will grow regardless of initial radius.
\end{abstract}

electric ion, condensation nucleus, droplet growth, non-dimensional charge, critical radius

Citation: Guo P S, Liu H L. Effect of charge on growth of droplets with ionic condensation nuclei. Chinese Sci Bull, 2011, 56: 1942-1946, doi: 10.1007/s11434$010-4261-6$

In many systems, vapor condensation is the main process of the heat and mass transfer with phase change. To enhance or control such processes, the vapor condensation process must be well studied. So far, only conventional studies on the heat or mass transfers have been conducted [1]. For example, to study passive condensation heat-transfer and mass-transfer enhancement, researchers mainly analyze the effect of geometry on the vapor condensation system [2-5]. Studies on active condensation heat transfer and mass transfer enhancement have focused on the effect of the condensation-surface fluctuations produced by external fields [6-9]. In addition, there are some studies on the effect of condensation-surface tension [10-12]. Vapor condensation enhancement is important not only in the process of the heat and mass transfer with phase change but also in drought relief applications. Vapor condensation occurs through the nucleation of tiny droplets, which gradually grow. The factors that affect droplet growth have attracted growing attention. A classical thermodynamics treatment

*Corresponding author (email: gpingsheng@263.net) using phase-equilibrium analysis suggests that the tiny droplets in the vapor must have an initial critical radius to grow $[13,14]$. If the initial droplets were formed only through fluctuations, the initial droplets will be generally too tiny to grow. Thus the initial critical radius affects droplet growth, which is a geometric factor. The magnitude of the mass transfer is dependent on the magnitude of some geometric factors. However it is important to investigate other type of factors that affect droplet growth. Recently, researchers have observed that the presence of external energy fields can enhance or weaken mass transfer [15-17]. The chemical potential drives mass transfer and is dependent not only to the temperature and pressure of the system, but also to the presence of external energy fields. Therefore, external energy fields may enhance or control mass transfer. There have been studies on the enhancement and control of heat and mass transfer by using the electric field [6-9]. However, there have been no studies on the effect of charge on the growth of droplets with an ionic condensation nucleus. In this paper, we explore the effect of charge on the growth of droplets with an ionic nucleus. This has the 
potential to provide definite guidance for the development of new techniques for enhancing the heat and mass transfer and for increasing the efficacy of drought relief applications.

\section{General form of chemical potential}

\subsection{Classical chemical potential}

Because the mass change during droplet growth may result in an energy change in this thermodynamic system, it is useful to define a chemical potential. For our system, the differential expression of Gibbs free energy is $\mathrm{d} G=-S \mathrm{~d} T+$ $V \mathrm{~d} p+\sum \mu_{k} \mathrm{~d} n_{k}$, where $\mu_{k}$ is

$$
\mu_{k}=\left(\partial G / \partial n_{k}\right)_{T, p, n_{j}} .
$$

Eq. (1) is the partial molar Gibbs free energy of component $k$ under constant temperature and pressure, which is the chemical potential of the system. According to basic thermodynamic equations, $\Sigma \mu_{k} \mathrm{~d} n_{k}$ also appears in the differential expressions of other basic thermodynamic quantities:

$$
\left\{\begin{array}{l}
\mathrm{d} U=T \mathrm{~d} S-p \mathrm{~d} V+\sum \mu_{k} \mathrm{~d} n_{k}, \\
\mathrm{~d} H=T \mathrm{~d} S+V \mathrm{~d} p+\sum \mu_{k} \mathrm{~d} n_{k}, \\
\mathrm{~d} F=-S \mathrm{~d} T-p \mathrm{~d} V+\sum \mu_{k} \mathrm{~d} n_{k},
\end{array}\right.
$$

where $U$ is the systemic internal energy, $H$ is the systemic enthalpy, and $F$ is the systemic free-energy. Using the above differential expressions, the chemical potential is also defined as $\mu_{k}=\left(\partial U / \partial n_{k}\right)_{S, V, n j}, \mu_{k}=\left(\partial H / \partial n_{k}\right)_{S, p, n_{j}}$ and $\mu_{k}=(\partial F /$ $\left.\partial n_{k}\right)_{T, V, n j},(j \neq k)$. Thus there are four definitions of chemical potential. Although there are different chemical-potential forms, they are equivalent. Due to the fact that many practical problems are studied under constant temperature and pressure, this paper uses the chemical-potential definition that is based on the Gibbs free energy.

\subsection{Generalization of the chemical potential}

In the absence of external energy fields, the Gibbs free energy is defined as $G=U-T S+p V$, where $U$ is the internal energy. However, it is normal to include not only the internal energy of the system, but also the energy stored in external fields. We define the generalized $U^{\prime}$ as $U^{\prime}=U+\Sigma W_{i}$, where $W_{i}$ is the energy stored in the $i$ th external field of the system. Thus the general Gibbs free energy, $G^{\prime}$, is defined as

$$
G^{\prime}=U^{\prime}-T S+p V=G+\sum W_{i} .
$$

Correspondingly the chemical-potential of component $k$ is generalized as

$$
\mu_{k}^{\prime}=\left(\partial G^{\prime} / \partial n_{k}\right)_{T, p, n_{j}}=\left(\partial G / \partial n_{k}+\frac{\partial}{\partial n_{k}} \sum W_{i}\right)_{T, p, n_{j}} .
$$

Using eq. (1), the general definition, eq. (3), of chemical potential can be simplified to

$$
\mu_{k}^{\prime}=\mu_{k}+\left(\frac{\partial}{\partial n_{k}} \sum W_{i}\right)_{T, p, n_{j}},(j \neq k),
$$

where the system is kept under constant temperature, pressure, component concentration, and external fields.

\section{Droplet-growth kinetics}

The droplet growth is a process of mass transfer with phase change. For the purposes of this investigation, we will assume constant pressure and temperature and the presence of ionic condensation nuclei. In addition, we will refer to the liquid and vapor phases as the $\alpha$ and $\beta$ phases, respectively. The presence of electric fields necessitates using the general form of the chemical potential. When computing the electric field energy, we assume the electric ions are apart very far from each other. Therefore, each ion behaves as if it existed alone. This will simplify the form of the generalized chemical potential, and we need only research the kinetics of the growth of a single droplet with an ionic condensation nucleus. Let $\mu_{\alpha}^{\prime}$ and $\mu_{\beta}^{\prime}$ express the chemical potential of the $\alpha$ phase and $\beta$ phase, respectively, and that the ion is a sphere conductor of radius $a$ and charge $q$. The droplet has radius $r$ and all the vapor is outside the droplet. Thus, there are three electric displacement densities:

$$
\left\{\begin{array}{l}
D=E=0,(R<a), \\
D_{\alpha}=\varepsilon E_{\alpha}=q /\left(4 \pi R^{2}\right),(a<R<r), \\
D_{\beta}=\varepsilon_{0} E_{\beta}=q /\left(4 \pi R^{2}\right),(R>r) .
\end{array}\right.
$$

Therefore, the electric field energies of the $\alpha$ and $\beta$ phases are

$$
\left\{\begin{array}{l}
W_{\alpha}=\frac{1}{2} \int_{a}^{r} \frac{q^{2} \mathrm{~d} R}{4 \pi \varepsilon R^{2}}=-\frac{q^{2}}{8 \pi \varepsilon r}+\frac{q^{2}}{8 \pi \varepsilon a} \\
W_{\beta}=\frac{1}{2} \int_{r}^{\infty} \frac{q^{2} \mathrm{~d} R}{4 \pi \varepsilon_{0} R^{2}}=\frac{q^{2}}{8 \pi \varepsilon_{0} r}
\end{array}\right.
$$

Let $p$ be the saturated vapor pressure when the boundary surface between the vapor and liquid is flat. In the absence of the ion, the phase equilibrium condition is

$$
\mu_{\alpha}(T, p)=\mu_{\beta}(T, p) .
$$

In the case of a spherical boundary surface between vapor and droplet, let $p^{\prime}$ be the vapor pressure of the $\beta$ phase. In the absence of the ion, due to the equilibrium of the two-phase pressure, the pressure of the $\alpha$ phase is $p^{\prime}+2 \sigma / r$, where $\sigma$ is surface tension coefficient. Thus, when the boundary surface between vapor and droplet is spherical, 
the chemical potential of the $\alpha$ and $\beta$ phase in the absence of the ion are $\mu_{\alpha}\left(T, p^{\prime}+2 \sigma r\right)$ and $\mu_{\beta}\left(T, p^{\prime}\right)$, respectively. Next we will explore the situation that involves the boundary surface being spherical and the presence of an ionic nucleus. According to the general definition, eq. (4), of chemical potential and the mass differential relation, $\partial n_{\alpha}=-\partial n_{\beta}$, of the $\alpha$ and $\beta$ phases, the chemical potentials become

$$
\left\{\begin{array}{l}
\mu_{\alpha}^{\prime}\left(T, p^{\prime}+\frac{2 \sigma}{r}\right)=\mu_{\alpha}\left(T, p^{\prime}+\frac{2 \sigma}{r}\right)+\frac{q^{2}}{32 \varepsilon \pi^{2} r^{4}} v_{\alpha}^{0}, \\
\mu_{\beta}^{\prime}\left(T, p^{\prime}\right)=\mu_{\beta}\left(T, p^{\prime}\right)+\frac{q^{2}}{32 \varepsilon_{0} \pi^{2} r^{4}} v_{\alpha}^{0} .
\end{array}\right.
$$

where $V=4 \pi r^{3} / 3, v_{\alpha}^{0}=\left(\partial V / \partial n_{\alpha}\right)_{p^{\prime}, T}$, and $v_{\alpha}^{0}$ is the partial molar volume. If the vapor is treated as an ideal gas, the chemical potential, $\mu_{\beta}\left(T, p^{\prime}\right)$, of the $\beta$ phase with a spherical boundary surface may be expressed as [13]

$$
\mu_{\beta}\left(T, p^{\prime}\right)=\mu_{\beta}(T, p)+\mathrm{R} T \ln \left(p^{\prime} / p\right) .
$$

Next we consider that changes in the liquid properties are not obvious when the pressure changes. We expand $\mu_{\alpha}\left(T, p^{\prime}+2 \sigma / r\right)$ in a series and keep the linear term, which yields

$$
\mu_{\alpha}\left(T, p^{\prime}+\frac{2 \sigma}{r}\right)=\mu_{\alpha}(T, p)+\left(p^{\prime}-p+\frac{2 \sigma}{r}\right) v_{\alpha}^{0} .
$$

Substituting eqs. (7), (8) into eq. (6) yields

$$
\left\{\begin{array}{l}
\mu_{\alpha}^{\prime}=\mu_{\alpha}(T, p)+\left(p^{\prime}-p+\frac{2 \sigma}{r}+\frac{q^{2}}{32 \pi^{2} \varepsilon r^{4}}\right) v_{\alpha}^{0}, \\
\mu_{\beta}^{\prime}=\mu_{\beta}(T, p)+\mathrm{R} T \ln \frac{p^{\prime}}{p}+\frac{q^{2}}{32 \pi^{2} \varepsilon_{0} r^{4}} v_{\alpha}^{0} .
\end{array}\right.
$$

According to non-equilibrium thermodynamic theory, the linear mass transport of component $i$ is

$$
J_{i}=\sum_{k}^{N} L_{i k} X_{k},
$$

where $X_{k}$ is thermodynamic force, $J_{i}$ is thermodynamic current of the mass transport of component $i$ and $L_{i k}$ is a phenomenological coefficient. In this system, there is only one kind of component, and the thermodynamic force is determined by only the chemical-potential difference. Therefore, eq. (10) simplifies to

$$
J=L\left[\mu_{\beta}^{\prime}-\mu_{\alpha}^{\prime}\right],
$$

where $J$ is the mass transfer from the $\alpha$ phase to the $\beta$ phase per unit time and per unit area. Substituting eqs. (5) and (9) into eq. (11) yields

$$
\begin{aligned}
J & =L\left[\mu_{\beta}^{\prime}-\mu_{\alpha}^{\prime}\right]=L\left[\mathrm{R} T \ln \frac{p^{\prime}}{p}\right. \\
& \left.-\left(p^{\prime}-p+\frac{2 \sigma}{r}\right) v_{\alpha}^{0}+\frac{q^{2}}{32 \pi^{2} r^{4}}\left(\frac{1}{\varepsilon_{0}}-\frac{1}{\varepsilon}\right) v_{\alpha}^{0}\right] .
\end{aligned}
$$

Using $J=\frac{\mathrm{d} m}{S \mathrm{~d} t}=\frac{\mathrm{d}\left(4 \pi\left(r^{3}-a^{3}\right) \rho / 3\right)}{4 \pi r^{2} \mathrm{~d} t}=\rho \frac{\mathrm{d} r}{\mathrm{~d} t}$, where $\rho$ is liquid density, eq. (12) can be expressed as

$$
\begin{aligned}
\rho \frac{\mathrm{d} r}{\mathrm{~d} t}= & L\left[\mathrm{R} T \ln \frac{p^{\prime}}{p}-\left(p^{\prime}-p+\frac{2 \sigma}{r}\right) v_{\alpha}^{0}\right. \\
& \left.+\frac{q^{2}}{32 \pi^{2} r^{4}}\left(\frac{1}{\varepsilon_{0}}-\frac{1}{\varepsilon}\right) v_{\alpha}^{0}\right],
\end{aligned}
$$

which is the droplet-growth kinetics equation in the presence of an ionic condensation nucleus

\section{Effect of charge on droplet growth}

\subsection{Droplet-growth kinetics curves}

To study the effect of charge on the growth of droplets with an ionic condensation nucleus, the droplet growth kinetics eq. (13) must be simplified. First, $p^{\prime}-p<<2 \sigma r, p^{\prime}-p$ may be neglected [14] in eq. (13) which gives

$$
\begin{aligned}
& \rho \frac{\mathrm{d} r}{\mathrm{~d} t}=L\left(\mathrm{R} T \ln \frac{p^{\prime}}{p}\right) \\
& \times\left[1-\left(2 \sigma v_{\alpha}^{0} /\left(r \mathrm{R} T \ln \frac{p^{\prime}}{p}\right)\right)\right. \\
& \left.+\frac{q^{2}}{64 \sigma \pi^{2} r^{4}}\left(\frac{1}{\varepsilon_{0}}-\frac{1}{\varepsilon}\right)\left(2 \sigma v_{\alpha}^{0} /\left(\mathrm{R} T \ln \frac{p^{\prime}}{p}\right)\right)\right] .
\end{aligned}
$$

Then, let $r_{c}=\left(2 \sigma v_{\alpha}^{0} /\left(R T \ln \left(p^{\prime} / p\right)\right)\right)$, where $r_{\mathrm{c}}$ is the critical droplet radius [13]. Thus, we may define a non-dimensional droplet radius $y=r / r_{\mathrm{c}}$, a non-dimensional time $x=2 L \sigma v_{\alpha}^{0} t /$ $\left(\rho r_{\mathrm{c}}^{2}\right)$, and a non-dimensional charge $\theta=q^{2}\left(1 / \Sigma_{0}-1 / \varepsilon\right) /$ $\left(64 \sigma \pi^{2} r_{\mathrm{c}}^{3}\right)$. Substituting into eq. (14) yields

$$
\frac{\mathrm{d} y}{\mathrm{~d} x}=\left[1-\frac{1}{y}+\frac{\theta}{y^{4}}\right] \text {. }
$$

when $\theta=0$, eq. (15) becomes the droplet growth kinetics equation in the absence of an ionic nucleus. To further explore the dynamics of the system, we may vary the non-dime- nsional parameter $\theta$. let $y_{0}$ and $y_{\mathrm{c}}$ represent the non-dimensional initial droplet radius $\left(x=0, y=y_{0}\right)$ and non-dimensional critical droplet radius, respectively.

\subsection{Results}

As shown in Figure 1, $y_{\mathrm{c}}=1$ is critical droplet growth point. When $y_{0}>1$, the droplet grows. When $y_{0}<1$, the droplet decreases in size until it vanishes. Thus, $y_{\mathrm{c}}=1$ is the non-dimensional critical radius in the absence of a charged nucleus. Figures 2-6 show that when an ionic condensation nucleus is present, the non-dimensional critical radius of 
droplet growth is less than that of when the ion is absent, This behavior can be seen for values, $\theta=0.01$ and $0.98<y_{\mathrm{c}}<0.99<1$. This is the reason that droplet growth is enhanced with an ionic condensation nucleus. Further analysis indicates that $y_{\mathrm{c}}$ decreases as $\theta$ increases. This behavior can be seen for values, $\theta=0.1$ and $0.81<y_{c}<0.82$. This can be seen in the progression of $y_{\mathrm{c}}$ and $\theta$ in Figures 2-6. Furthermore, when the ionic nucleus is present, rather than vanishing, droplets with an initial radius less than $y_{\mathrm{c}}$ shrink to a steady state value, $y_{0}^{\prime \prime}$. This behavior, which is different from that without the ionic nucleus, can be seen for values, $\theta=0.01$ and $y_{0}^{\prime \prime} \approx 0.23$. This value, $y_{0}^{\prime \prime}$, also increases as $\theta$ increases. When the initial droplet radius is less than both $y_{\mathrm{c}}$ and $y_{0}^{\prime \prime}$, the droplet grows until its radius reaches $y_{0}^{\prime \prime}$. This behavior can be seen in Figure 5. For $y_{0}=0.6$, $y_{0}=0.1$, and $y_{0}=0.01$, the $y$ increases to a steady-state value of 0.67 . Thus, when an ionic condensation nucleus is present, although the initial droplet radius may be small $\left(y_{0}<y_{0}^{\prime \prime}\right)$, the droplet radius also continuously increases to a steady-state value $y_{0}^{\prime \prime}$. This is also the reason that the droplet growth is enhanced. Using the above analysis, it can be seen that when $0<y_{0}<y_{0}^{\prime \prime}$, the droplet radius increases to a steady-state value $y_{0}^{\prime \prime}$, when $y_{0}^{\prime \prime}<y_{0}<y_{\mathrm{c}}$, the drop radius will decrease to $y_{0}^{\prime \prime}$, and when $y_{0}>y_{\mathrm{c}}$, the droplet will grow without bound.

As $\theta$ increases, $y_{\mathrm{c}}$ decreases and $y_{0}^{\prime \prime}$ increases. Therefore, there is a critical value of $\theta$ that leads to $y_{0}^{\prime \prime} \geqslant y_{\mathrm{c}}$
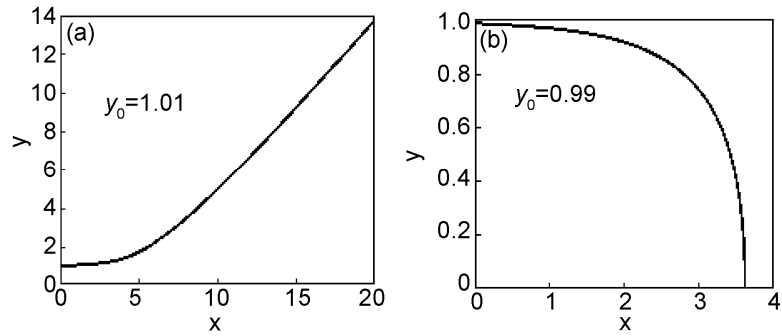

Figure 1 Temporal evolution of the non-dimensional radius, where the non-dimensional charge, $\theta=0$. The plot shows $y$ vs. $x$, which is the non-dimensional time coordinate.
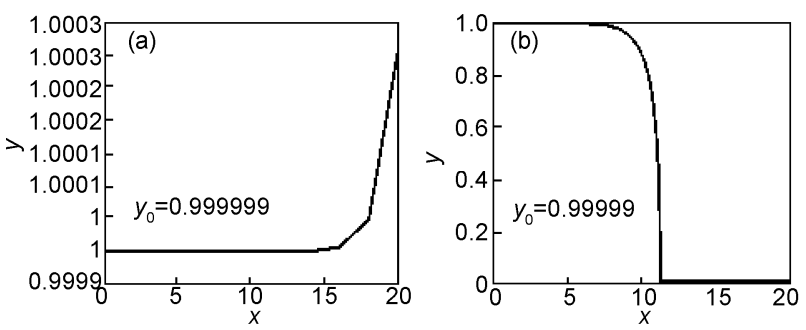

Figure 2 Temporal evolution of the non-dimensional radius, where the non-dimensional charge, $\theta=0.000001$. The plot shows $y$ vs. $x$, which is the non-dimensional time coordinate. which means that the region where the droplet radius decreases, $y_{0}^{\prime \prime}<y_{0}<y_{\mathrm{c}}$, no longer exists. When $\theta$ reaches this
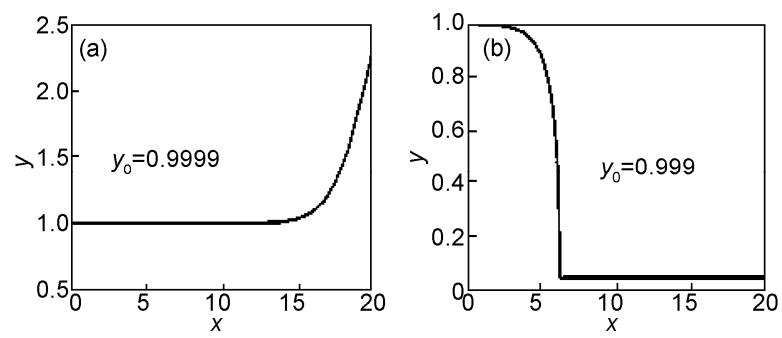

Figure 3 Temporal evolution of the non-dimensional radius, where the non-dimensional charge, $\theta=0.0001$. The plot shows $y$ vs. $x$, which is the non-dimensional time coordinate.
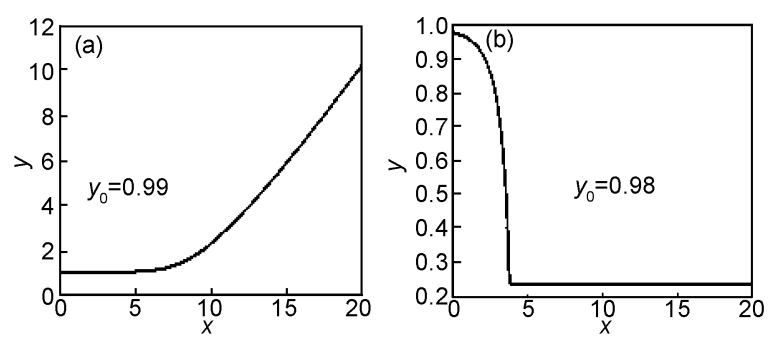

Figure 4 Temporal evolution of the non-dimensional radius, where the non-dimensional charge, $\theta=0.01$. The plot shows $y$ vs. $x$, which is the non-dimensional time coordinate.
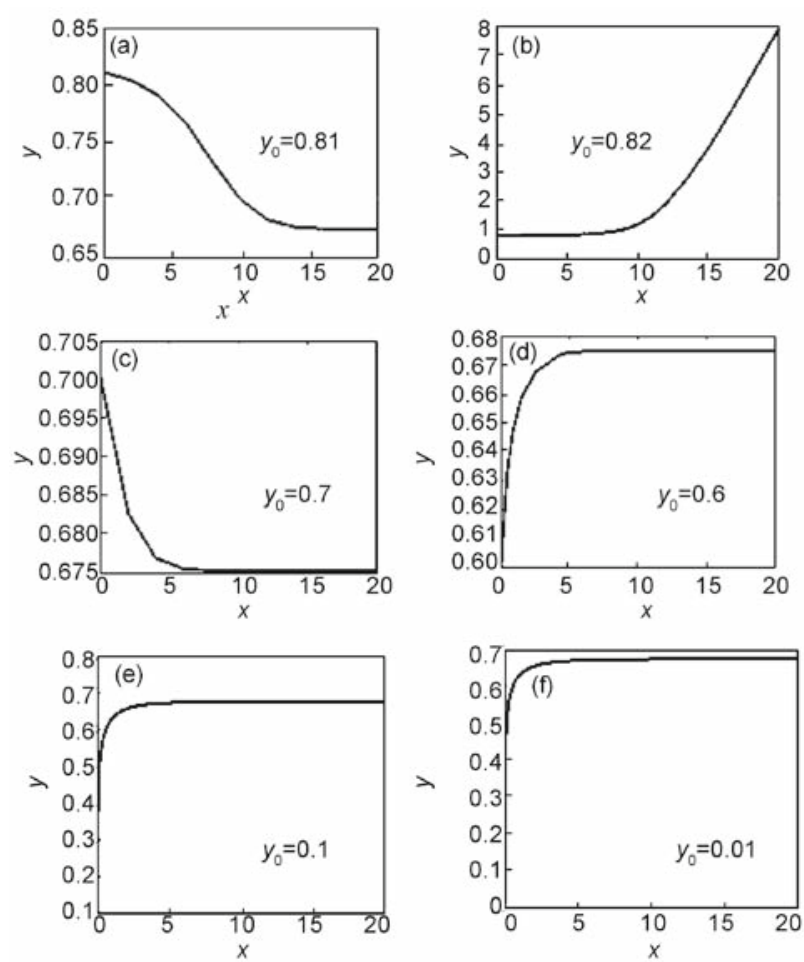

Figure 5 Temporal evolution of the non-dimensional radius, where the non-dimensional charge, $\theta=0.1$. The plot shows $y$ vs. $x$, which is the non-dimensional time coordinate. 

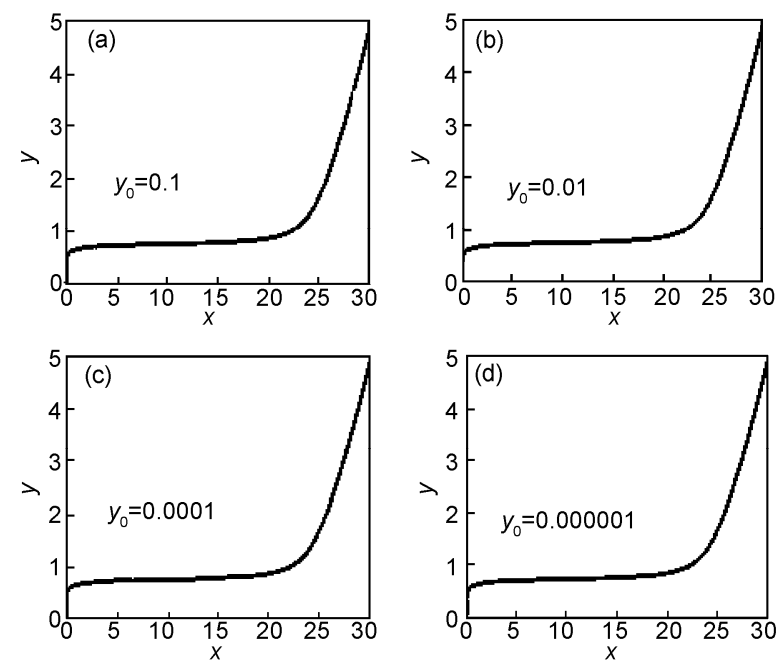

Figure 6 Temporal evolution of the non-dimensional radius, where the non-dimensional charge, $\theta=0.107$. The plot shows $y$ vs. $x$, which is the non-dimensional time coordinate.

value, droplets of arbitrarily small radius will grow. This is shown in Figure 6 for $\theta=0.107$, which shows growth for all computed values $\left(y_{0}=0.1, y_{0}=0.01, y_{0}=0.0001, y_{0}=0.000001\right)$. Therefore, above the critical value of $\theta$, droplet growth is independent of the initial droplet radius. This shows that the greater the value of $\theta$, the greater the effect produced by the ionic nucleus. In electrical storm conditions, charged particles are common, which leads to widespread droplet growth. In a Wilson cloud chamber, the motion of ions is visible due to this charged-condensation-nucleus droplet growth behavior. This is due to the fact that traveling ions create more ions in their wake, which become condensation nuclei, which highlights the path of the original ion.

\section{Conclusions}

We used the general form of the chemical potential and the kinetic equation of mass transfer and established a growthkinetics model for droplets with ionic condensation nuclei. We then used assumptions to simplify the model. Using this model, we explored the effects of charge on droplet growth. For various values of $\theta$ droplet-growth kinetics curves were drawn. We showed that the critical radius for ionic-condensation-nucleus-droplet growth is less than that of those lacking an ionic nucleus. Furthermore, the results indicated that in the presence of an ionic nucleus, a droplet of radius less than the critical radius will not vanish, and instead will approach a steady-state radius. We showed that as the non- dimensional charge, $\theta$, increases, the non-dimensional critical radius for the droplet growth decreases and the corresponding steady-state value of the droplet radius increases. When the non-dimensional charge increases to the critical value, droplets will grow for arbitrarily small radius. Thus, in practice, the vapor condensation process may be enhanced and controlled by adding charged particles.

This work was supported by the National Basic Research Program of China (2000026307).

1 Butrymowicz D, Trela M, Karwacki J A. Enhancement of condensation heat transfer by means of passive and active condensate drainage techniques. Int J Refrig, 2003, 26: 473-484

2 Chen Y P, Wu J F, Shi M H, et al. Numerical simulation for steady annular condensation flow in triangular microchannels. Int Comm Heat Mass Transfer, 2008, 35: 805-809

3 Stefano N, John R T. Numerical modeling of laminar annular film condensation for different channel shapes. Int J Heat Mass Transfer, 2010, 53: 2615-2627

4 Akhavan-Behabadi M A, Ravi K, Mohseni S G. Condensation heat transfer of R-134a inside a microfin tube with different tube inclinations. Int J Heat Mass Transfer, 2007, 50: 4864-4871

5 Mua C F, Pang J J, Lu Q Y, et al. Effects of surface topography of material on nucleation site density of dropwise condensation. Chem Eng Sci, 2008, 63: 874-880

6 Bologa M K, Korovkin V P, Savin I K. Mechanism of condensation heat transfer enhancement in an electric field and the role of capillary processes. Int J Heat Mass Transfer, 1995, 38: 175-182

7 Suriyan L, Paisarn N, Somchai W. A review of electrohydrodynamic enhancement of heat transfer. Renew Sustain Energy Rev, 2008, 11: 858-876

8 Butrymowic Z D, Trei A M, Karwacki J. Enhancement of condensation heat transfer by means of EHD condense drainage. Int $\mathrm{J}$ Therm Sci, 2002, 41: 646-657

9 Yabe A, Mori Y, Hijikata K. Active heat transfer enhancement by utilizing electric fields. Annual Rev Heat Transfer, 1997, 7: 193-244

10 Rose J W. Surface tension effects and enhancement of condensation heat transfer. Chem Eng Res Des, 2004, 82: 419-429

11 Chang T B, Wen Y Y. Effects of uniform suction and surface tension on laminar filmwise condensation on a horizontal elliptical tube in a porous medium. Int J Therm Sci, 2009, 48: 2323-2330

12 Xin R C, Awwad A, Dong Z F, et al. Experimental study of single phase and two phase flow in annular helicoidar pipes. ASME, Heat Transfer Div, 1996, 330: 11-17

13 Wang Z C. Thermodynamic Statistical Physics (in Chinese). Beijing: Higher Education Press, 2003. 126-127

14 Wang Z X. Thermodynamics (in Chinese). Beijing: Peking University Press, 2005. 186-187

15 Chen Q, Meng J A. Field synergy analysis and optimization of the convective mass transfer in photocatalytic oxidation reactors. Int $\mathbf{J}$ Heat Mass Transfer, 2008, 51: 2863-2870

16 Lei Z, Wu Y Y, Lu S Q, et al. Mass Transfer modeling in pervaporation based on multi-fields synergy theory. Chin J Chem Eng, 2008, 16: 79-83

17 Guo P S, Han G Z, Zhang N, et al. Mechanism and fields synergism analysis of desorption enhancement by ultrasonic field (in Chinese). $\mathrm{J}$ Chem Eng Chin Univ, 2006, 20: 300-305

Open Access This article is distributed under the terms of the Creative Commons Attribution License which permits any use, distribution, and reproduction in any medium, provided the original author(s) and source are credited. 